\title{
Quantification of spiral artery remodelling using an Adobe Photoshop-based technique
}

\author{
H A Otun, B A Innes, G E Lash ${ }^{\dagger}$, B Schiessl ${ }^{\dagger+}$, E Ball, R F Searle, S C Robson and J N Bulmer \\ Reproductive and Vascular Biology Group, Institute of Cellular Medicine, Newcastle University, Newcastle upon \\ Tyne, UK \\ Correspondence should be addressed to J N Bulmer; Email: judith.bulmer@ncl.ac.uk \\ ${ }^{+}$(G E Lash is now at Guangzhou Institute of Pediatrics, Guangzhou Women and Children's Medical Center, Guangzhou, China) \\ ${ }^{+\dagger}$ (B Schiessl is now at Praxis für Pränatalmedizin, Munchen, Germany) *(E Ball is now at Obstetrics and Gynaecology, Barts \\ NHS trust, London, UK)
}

\begin{abstract}
Uterine spiral arteries undergo remodelling in normal pregnancy, with replacement of the musculoelastic arterial media by fibrinoid containing extravillous trophoblast cells. Deficient spiral artery remodelling is associated with several adverse pregnancy outcomes. Although there are distinct components of spiral artery remodelling, assessment is subjective and often based on an overall impression of morphology. We aimed to develop a quantitative approach for assessment of uterine spiral artery remodelling. Placental bed biopsies were immunostained using smooth muscle markers, digital images of spiral arteries were captured and Adobe Photoshop was used to analyse positive immunostaining. The method was then used to investigate variation in the same vessel at different levels within a paraffin block, and the effect of parity, pre-eclampsia or miscarriage on vascular smooth muscle cell content. Results were also compared with a more subjective morphology-based assessment system. There was good intra- and interobserver agreement and the method correlated well with the more subjective assessment system. There was an overall reduction in vascular smooth muscle, as detected by caldesmon 1 (h-caldesmon) immunopositivity, with increasing gestational age from 8 weeks to term. A previous pregnancy did not affect the amount of spiral artery smooth muscle. Comparison of pre-eclampsia and late miscarriage samples with controls of the appropriate gestational age demonstrated increased medial smooth muscle in pathological samples. This technique provides a simple, rapid, reproducible and inexpensive approach to quantitative assessment of spiral artery remodelling in normal and pathological human pregnancy, a process which although fundamental for successful pregnancy, is still incompletely understood. Reproduction (2019) 158 389-397
\end{abstract}

\section{Introduction}

Remodelling of uterine spiral arteries (SpAs) in decidua and superficial myometrium is critical for successful human pregnancy. Failure of this process, particularly in superficial myometrial segments, is associated with pregnancy complications such as pre-eclampsia, fetal growth restriction, preterm labour and second trimester miscarriage (Khong et al. 1986, Pijnenborg et al. 1991, Kim et al. 2003, Ball et al. 2006a).

The histological features of SpA remodelling are well described with initial vascular dilatation, separation of medial vascular smooth muscle cells (VSMCs) and endothelial cell (EC) swelling in the absence of trophoblast, termed 'decidua-associated remodelling' (Craven et al. 1998, Pijnenborg et al. 2006). Extravillous trophoblast (EVT) cells migrate within the lumen of the SpAs against the direction of blood flow and attach to the wall, with transient loss of ECs. Ultimately there is complete loss of VSMCs and elastic fibres and replacement by amorphous fibrinoid material containing intramural EVT cells (reviewed in Pijnenborg et al. 2006).

Assessment of SpA remodelling is often based on an overall impression of histological features. Vessels which lack features of this process are described as 'non-remodelled', whereas in 'partially remodelled' SpAs there may be some loss of VSMCs, some fibrinoid deposition, and endovascular and/or intramural EVT cells may be detected. 'Completely remodelled' SpAs show replacement of the medial elastic and VSMCs by fibrinoid incorporating intramural EVT cells. Categories for different stages of SpA remodelling have been proposed but these are subjective and consistency between observers is uncertain (Ball et al. 2006b, Pijnenborg et al. 2006, Smith et al. 2009, Lash et al. 2016). Previous studies have used image analysis tools to quantify SpA remodelling (Starzyk et al. 1997, Morgan et al. 1999), but these methods have not been widely 
employed and a simple, relatively non-time consuming and reproducible technique is required.

It is established that pre-eclampsia is associated with failed remodelling of myometrial segments of SpAs (Pijnenborg et al. 1991), but this is unlikely to be an 'all or nothing' phenomenon since Meekins et al. (1994) reported trophoblast invasion in 18\% of myometrial segments in pre-eclampsia compared with $76 \%$ in normal pregnancy. Late miscarriage is also associated with failed $\mathrm{SpA}$ transformation with reduced endovascular and intramural trophoblast and less extensive fibrinoid in myometrial SpAs from late miscarriage compared with controls (Ball et al. 2006a). A standardised objective approach to assessment of the various components of spiral artery remodelling may improve the understanding of the failure of this process in pathological pregnancy.

Studies of SpA structure in humans have focused on non-pregnant endometrium (Abberton et al. 1996, 1999, Rogers \& Abberton 2003). As the SpAs develop the VSMCs exhibit differing expression of alpha smooth muscle actin ( $\alpha \mathrm{SMA}$; actin alpha 2 (ACTA2)), calponin, heavy chain (h-) caldesmon (caldesmon 1, CALD1), smooth muscle alpha tropomyosin, gamma SMA, desmin and myosin heavy chain. Although there are some discrepancies between studies, $\alpha \mathrm{SMA}$ is expressed early in SpA differentiation (Skalli et al. 1986, Owens 1995), whereas desmin, h-caldesmon (h-cal) and myosin heavy chain $(\mathrm{MyHC})$ are expressed by differentiated VSMCs (Price et al. 1994, Owens 1995). Immunohistochemical studies of uterine SpAs in pregnancy have mainly used antibodies to $\alpha S M A$ and desmin, but there is no information regarding expression of different VSMC markers in SpAs in pregnancy.

The mechanism and time scale of involution of uteroplacental arteries following delivery is unclear, but pregnancy-induced morphological changes in SpAs are largely reversed post-partum. Pre-eclampsia occurs most commonly in primigravidae, with a lower incidence in subsequent pregnancies (Macgillivray 1958, Chesley 1984); incomplete reversal of pregnancyinduced morphological changes and resultant 'weakening' of SpAs could provide a morphological explanation for the reduction in incidence of preeclampsia in parous women. In studies of non-pregnant hysterectomies, Khong et al. (2003) demonstrated increasing fragmentation of the internal elastic lamina of SpAs with increasing parity, providing some support for this proposal.

Despite the availability of image analysis techniques (Lehr et al. 2001, Matkowskyj et al. 2003), there have been few recent attempts to quantify SpA remodelling in human pregnancy. The aim of this study was to develop a simple, inexpensive and reproducible technique to quantify various morphological changes in SpA remodelling.

\section{Materials and methods}

\section{Subjects and tissue collection}

\section{Normal pregnancy samples}

Women undergoing elective surgical termination of apparently normal pregnancy or caesarean section were recruited at the Newcastle upon Tyne Hospitals NHS Foundation Trust, Newcastle upon Tyne. The study received approval from the Joint Ethics Committee of Newcastle and North Tyneside Health Authority and the University of Newcastle upon Tyne and all subjects gave informed written consent. Gestational age of first and second trimester samples was determined by ultrasound measurement of crown rump length or biparietal diameter immediately prior to the termination procedure. Placental bed biopsies were obtained as described detail previously (Robson et al. 2002), fixed within 30 min of sampling in $10 \%$ neutral-buffered formalin for $24 \mathrm{~h}$ and routinely processed into paraffin wax. Samples were initially assessed using immunohistochemistry to detect EVT (cytokeratin), endothelium (von Willebrand factor) and smooth muscle $(\alpha \mathrm{SMA})$ as previously described (Robson et al. 2002). All biopsies included in the study included decidua, myometrium, interstitial EVT and at least one SpA.

\section{Pathological pregnancy}

Placental bed biopsies were also obtained from pregnancies complicated by pre-eclampsia (gestational age 30-39 weeks) and from women undergoing evacuation of retained products of conception following late miscarriage (gestational age 13-19 weeks) (Lyall et al. 2001, Robson et al. 2002, Ball et al. 2006a). Placental bed biopsies from pathological pregnancies were snap frozen in liquid nitrogen-cooled isopentane within $10 \mathrm{~min}$ of sampling, stored sealed at $-80^{\circ} \mathrm{C}$. Adequacy of placental bed sampling was assessed by immunohistochemistry as for paraffin sections. Pathological samples were compared with similarly snap frozen placental bed biopsies from normal third trimester samples (gestational age 38-40 weeks) for the pre-eclampsia samples, and from normal early pregnancy (gestational age 13-19 weeks) for the late miscarriage samples.

\section{Immunohistochemistry}

Serial $3 \mu \mathrm{m}$ paraffin sections of normal pregnancy samples were immunostained using an avidin-biotin peroxidase method (Vectastain Elite mouse kit, Vector Laboratories, Peterborough, UK) as described previously (Robson et al. 2002, Ball et al. 2006b). Details of antibodies, clones, dilutions and pretreatments are shown in Table 1.

Paraffin sections were dewaxed in xylene and rehydrated in descending alcohol concentrations. Frozen sections were brought to room temperature. After a 10-min incubation in blocking serum, sections were incubated with primary antibody for $60 \mathrm{~min}$, biotinylated secondary antibody for $30 \mathrm{~min}$ and tertiary antibody for $30 \mathrm{~min}$ according to the kit instructions with two 5-min washes in $0.15 \mathrm{M}$ Tris buffered $0.05 \mathrm{M}$ saline (TBS; $\mathrm{pH}$ 7.6) between each step. All incubations were at room temperature. The reaction was developed using DAB 
Table 1 Primary antibodies used for immunohistochemistry.

\begin{tabular}{llcl}
\hline Antibody & Pre-treatment $^{\S}$ & Dilution & Clone \\
\hline h-caldesmon $^{*}$ & Citrate buffer pH 6.0 & $1: 50$ & h-CD \\
myosin heavy chain $^{\dagger}$ & Citrate buffer pH 6.0 & $1: 600$ & hsm-V \\
$\alpha$-smooth muscle actin & Trypsin pH 7.8 & $1: 75$ & $\alpha \mathrm{sm}-1$ \\
Desmin $^{\ddagger}$ & Trypsin pH 7.8 & $1: 50$ & DE-R-11 \\
Elastin $^{\ddagger}$ & Trypsin pH 7.8 & $1: 60$ & BA-4
\end{tabular}

*Dako; ${ }^{+}$Sigma Chemical Company; ${ }^{\ddagger}$ Novocastra Laboratories, Newcastle upon Tyne, UK; ${ }^{\S}$ Citrate buffer pre-treatments were pressure cooked for $1 \mathrm{~min}$; trypsin pre-treatment was at $37^{\circ} \mathrm{C}$ for $10 \mathrm{~min}$.

(3,3' diaminobenzidine tetrahydrochloride; Sigma Chemical Co.) containing $\mathrm{H}_{2} \mathrm{O}_{2}(0.03 \%)$ to give a brown reaction product. Frozen sections $(5 \mu \mathrm{m})$ of pathological pregnancy samples were immunostained with desmin (1:100) using an avidin biotin method (Vector Laboratories) as previously described (Robson et al. 2002).

All immunostained sections, apart from elastin, were counterstained with the Periodic Acid Schiff (PAS) technique to allow identification of fibrinoid and thereby facilitate localisation of remodelled spiral arteries. Sections were rinsed in water, incubated in 1\% Periodic acid (Sigma) for $5 \mathrm{~min}$, rinsed again in water, incubated in Schiff's reagent (Sigma) for $8 \mathrm{~min}$ and then rinsed again. All immunostained sections were counterstained with Mayer's haematoxylin, dehydrated, cleared and mounted in DPX (Sigma). Positive controls were included in each staining run. Negative controls in which primary antibody was omitted or substituted with non-immune mouse serum were performed for each specimen.

\section{Elastic van Gieson (EVG)}

Sections were incubated in potassium permanganate $(2 \%$ in water; Sigma) for $5 \mathrm{~min}$, rinsed in water and incubated in $5 \%$ oxalic acid (Sigma) for $1 \mathrm{~min}$. After a further tap water rinse, sections were rinsed with 95\% alcohol and incubated in Millers Elastin (VWR, Lutterworth, UK) for $2 \mathrm{~h}$. Excess stain was then removed with $95 \%$ alcohol. The sections were counterstained with van Gieson for 3 min, rinsed with 95\% alcohol, dehydrated in absolute alcohol, cleared in xylene and mounted in DPX.

\section{Image analysis using Adobe Photoshop}

Myometrial arteries were identified in sections immunostained to demonstrate VSMC. Although not all arteries were exact transverse sections, care was taken to select only arterial cross-sections that were symmetrical with a clearly visible lumen. Digital images of myometrial SpAs were captured from each immunostained section at $\times 200$ magnification using a Leica DM LA microscope coupled with a Hitachi HV-C20MA camera and a Personal Computer fitted with a Matrox analogue frame grabber. Images were saved in uncompressed TIFF format and analysed using Adobe Photoshop (Adobe Systems Incorporated).
Individual SpAs were identified (Fig. 1A) and the outer extent of the media determined from PAS and VSMC staining as previously described (Lash et al. 2016), selected using the magnetic lasso tool and copied to a new layer within the image. The luminal margin was similarly selected using the magnetic lasso tool and the luminal area was deleted from the image to leave an image only of the vessel wall (Fig. 1B). The total number of pixels within this image was noted. A positively stained area was then identified by eye and selected using the magic wand tool. Other immunopositive areas were then selected using the 'similar' command, with the threshold level set such that all positive areas but no negative areas were selected. This was determined by careful examination of the selected areas to ensure that no positive areas remained unselected, repeating this stage if necessary. For all antibodies, immunopositivity was uniform within the vessel wall with clear positive areas and minimal if any background reactivity. The immunopositive areas were then copied into a new layer (Fig. 1C). The number of pixels in the positive layer was noted and the positive pixel count in the immunostained sections was then expressed as a percentage of the total number of pixels in the vessel wall for each SpA segment (Fig. 1D). A further explanation of the method with screenshots is provided in Supplementary Fig. 1 (see section on supplementary data given at the end of this article).

\section{Statistical analysis}

Unless otherwise stated data are expressed as mean \pm standard error of mean (S.E.) \% positive pixels. Statistical analyses were performed using Minitab (Minitab Inc. Pennsylvania, USA). Statistical significance was determined using one-way ANOVA unless otherwise stated. Student's t-test was used for comparison between means. Linear regression analysis was used to assess the correlation between variables and nested ANOVA was used to assess variation between sections. Differences were considered statistically significant at $P<0.05$.

\section{Results}

\section{Comparison of anti-smooth muscle antibodies}

The technique was initially assessed in serial sections from normal placental bed biopsies at 10- to 12-week gestational age $(n=5)$. In each biopsy, five individual SpA cross-sections were identified in serial sections immunostained for h-cal, $\alpha \mathrm{SMA}$, desmin and MyHC to allow direct comparison of vascular smooth muscle quantification between the different antibodies. All analysis was by one investigator (HAO) blinded to the identity of the sections. Immunoreactivity was most abundant for h-cal (mean \% positive pixels $\pm \mathrm{SE}$, $46.3 \pm 7.0 \%$ ) with $\mathrm{MyHC}, \alpha \mathrm{SMA}$ and desmin all showing less positivity as assessed by positive pixels (h-cal $40.9 \pm 7.7 \%$; $\alpha \mathrm{SMA} 29.0 \pm 5.1 \%$; desmin $13.5 \pm 5.1 \%$ ) (Fig. 2). Immunostaining for h-cal was therefore used for subsequent studies. 


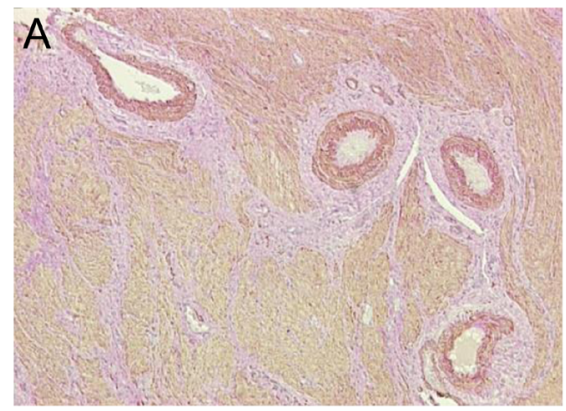

B

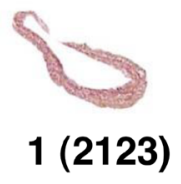

2 (2080)

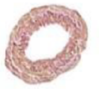

3 (1447)
C

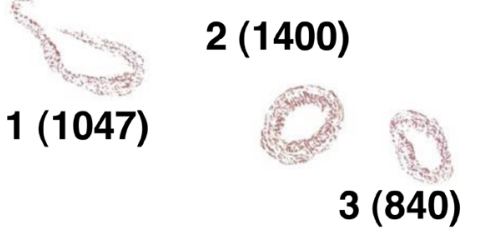

4 (1642)

4 (1004)

D

\begin{tabular}{|l|l|l|l|}
\hline $\begin{array}{l}\text { Vessel } \\
\text { Profile } \\
\text { number }\end{array}$ & Total Pixels & $\begin{array}{l}\text { Immunopositive } \\
\text { Pixels }\end{array}$ & $\%$ Immunopositive Pixels \\
\hline 1 & 2123 & 1047 & $49.3 \%$ \\
\hline 2 & 2080 & 1400 & $67.3 \%$ \\
\hline 3 & 1447 & 840 & $58.1 \%$ \\
\hline 4 & 1642 & 1004 & $61.1 \%$ \\
\hline
\end{tabular}

Figure 1 Schematic illustrating the photoshop method. (A) Section of placental bed biopsy immunostained for H-caldesmon and counterstained with PAS showing four cross-sections of a partially remodelled SpA. (B) Immunopositive SpA cross-sections were selected using the magnetic lasso tool and pasted into a new layer to give the total pixel number for each SpA wall cross section (numbers in parentheses). (C) The threshold intensity of immunostaining was identified using the magic wand tool, similar areas were selected and copied into a new layer to give the number of immunopositive pixels (numbers in parentheses). (D) Table showing the total and positive pixel numbers and calculation of \% positive pixels.

\section{Assessment of intraobserver and interobserver reproducibility}

Intraobserver reproducibility

An image of the same h-cal immunostained SpA was captured and analysed by one of the investigators (HAO) on ten separate occasions over a two week period. Positive pixels ranged from 49.08 to $55.87 \%$ with a median value of $53.83 \%$. The standard deviation of the analysis was 2.13 , representing $4.00 \%$ coefficient of variation.

\section{Interobserver variability}

Two observers (HAO and BS) independently captured and analysed images of the same ten individual SpAs immunostained for h-cal in placental bed biopsies at 8-12 weeks gestational age ( $n=7$ biopsies). Values from investigator 1 and investigator 2 for the ten SpAs investigated are shown in Fig. $3 \mathrm{~A}$ and $\mathrm{B}$. The $R^{2}$ value from linear regression analysis was 0.8744 , indicating very good agreement between the two independent observers. As can be seen in Fig. 3A, agreement was generally good, and there was no consistent pattern in discrepancy according to the number of positive pixels.

\section{Variation within spiral arteries}

Serial $3 \mu \mathrm{m}$ sections were cut from five placental bed samples, gestational age 13 weeks to term. The first and every subsequent fifth section were immunostained for h-cal with a total of six sections stained from each sample, representing a depth of $75 \mu \mathrm{m}$. For each sample five SpAs which extended through all the sections were identified for analysis and assessment by nested ANOVA. There was no significant difference in h-cal immunostaining over the total depth of the sections examined (Fig. 3C; $P=0.426$ ).

\section{Gestational age}

There was a progressive reduction in h-cal immunostaining with increasing gestational age: $8-10$ weeks: $55.49 \pm 7.18 \%$ ( $n=47$ SpAs, 6 biopsies); $12-14$ weeks: $42.52 \pm 8.05 \%$ ( $n=48$ SpAs, 6 biopsies); $16-20$ weeks: $36.22 \pm 3.97 \%(n=56$ SpAs, 7 biopsies); and $38-40$ weeks: $25.66 \pm 5.68 \%$ ( $n=37$ SpAs, 5 biopsies) (Fig. 4A). Although there were no significant differences between consecutive groups, the mean percentage positive pixel values was significantly reduced (ANOVA) at 16-20 

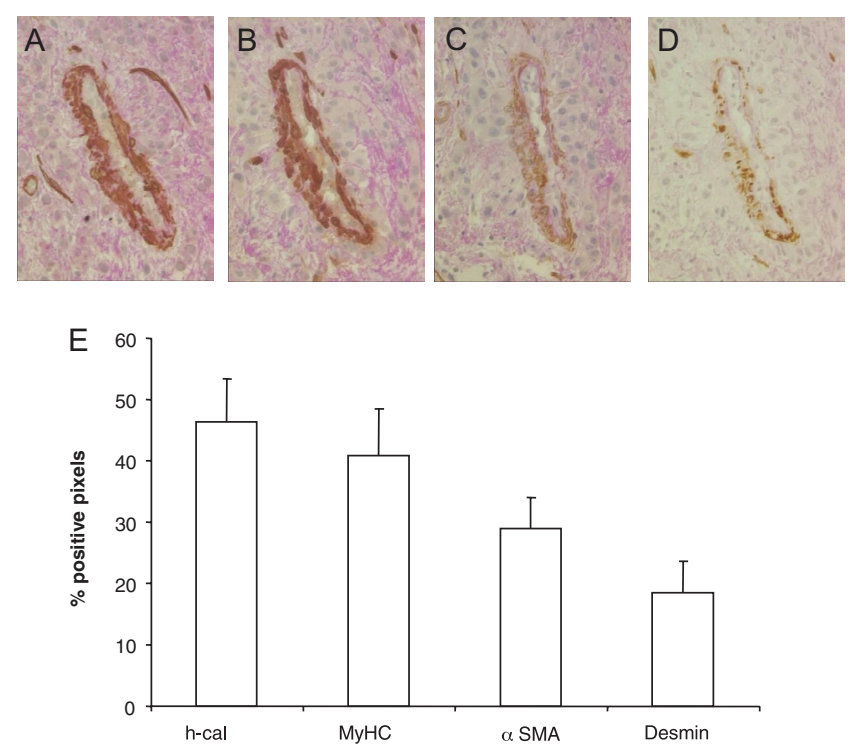

Figure 2 Serial sections of a partially remodelled SpA (magnification $\times 400$ ) showing immunostaining with: (A) h-caldesmon (h-cal); (B) myosin heavy chain (MyHC); (C) $\alpha$-smooth muscle actin $(\alpha \mathrm{SMA})$; (D) desmin. (E) Analysis of spiral artery smooth muscle immunostaining with h-cal, MyHC, $\alpha \mathrm{SMA}$ and desmin. Data are expressed as the percentage of positive pixels in the vessel wall image for each antibody ( $n=25$ SpAs from five placental bed samples).

weeks $(P=0.007, n=7)$ and $37-40$ weeks $(P=0.005$, $n=5)$ gestation compared with $8-10$ weeks gestation.

\section{Effect of parity}

In order to determine the effect of previous pregnancy on SpA smooth muscle content and elastin (assessed by immunostaining and EVG stain), myometrial arteries not associated with perivascular trophoblast were assessed in samples from ten nulliparous (gestational age 8-12 weeks; mean $9.2 \pm 0.33$ weeks) and ten multiparous women (gestational age 8-12 weeks; mean 9.9 \pm 0.41 weeks; mean parity $2.1 \pm 0.5$, range $1-6$ previous pregnancies) (Fig. 4B). A total of $100 \mathrm{SpAs}$ were examined by one investigator (HAO) who was blinded to the parity of the sample.

For both vascular smooth muscle and elastin there were no differences in the percentage of positive pixels in sections from nulliparous and multiparous women (h-cal: nulliparous $53.1 \pm 2.6 \%$, multiparous $48.5 \pm 2.5 \%$; elastin: nulliparous $20.4 \pm 2.6 \%$, multiparous $19.9 \pm 3.3 \%$; EVG: nulliparous $47.5 \pm 1.9 \%$, multiparous $49.4 \pm 1.6 \%$ ).

\section{Assessment of spiral artery transformation}

Assessment of 'partial remodelling' is subjective and includes a range of histological appearances. Two investigators (J N B and G E L) independently assessed histological features in 230 SpAs from 21 normal placental bed biopsies, including separation and
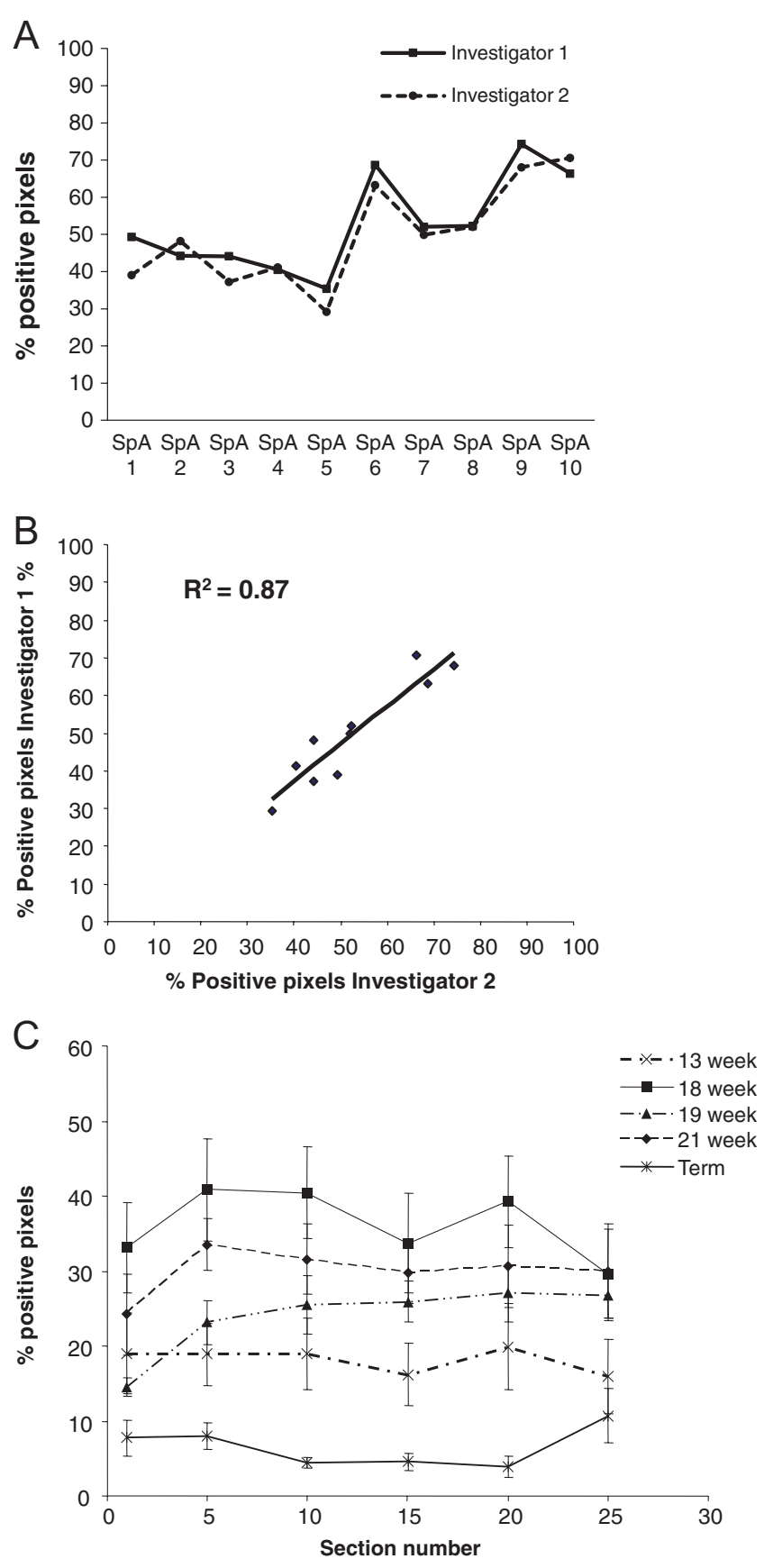

Figure 3 (A and B) Linear plot (A) and scatter plot (B) showing the percentage pixel counts for analyses carried out by two independent investigators ( $n=10$ spiral arteries in seven placental bed biopsies). (C) Variation in positive staining between $3 \mu \mathrm{m}$ step (1st, 5th, 10th, 15th, 20th, 25th) sections of individual spiral arteries in five different placental bed samples.

disruption of immunostained VSMCs, the extent of PASpositive fibrinoid, and the presence of endovascular and intramural trophoblast, detected by immunostaining for cytokeratin. SpAs were assigned to four groups based on subjective overall assessment of 'remodelling': non-remodelled $(N ; n=33)$, early partially remodelled 

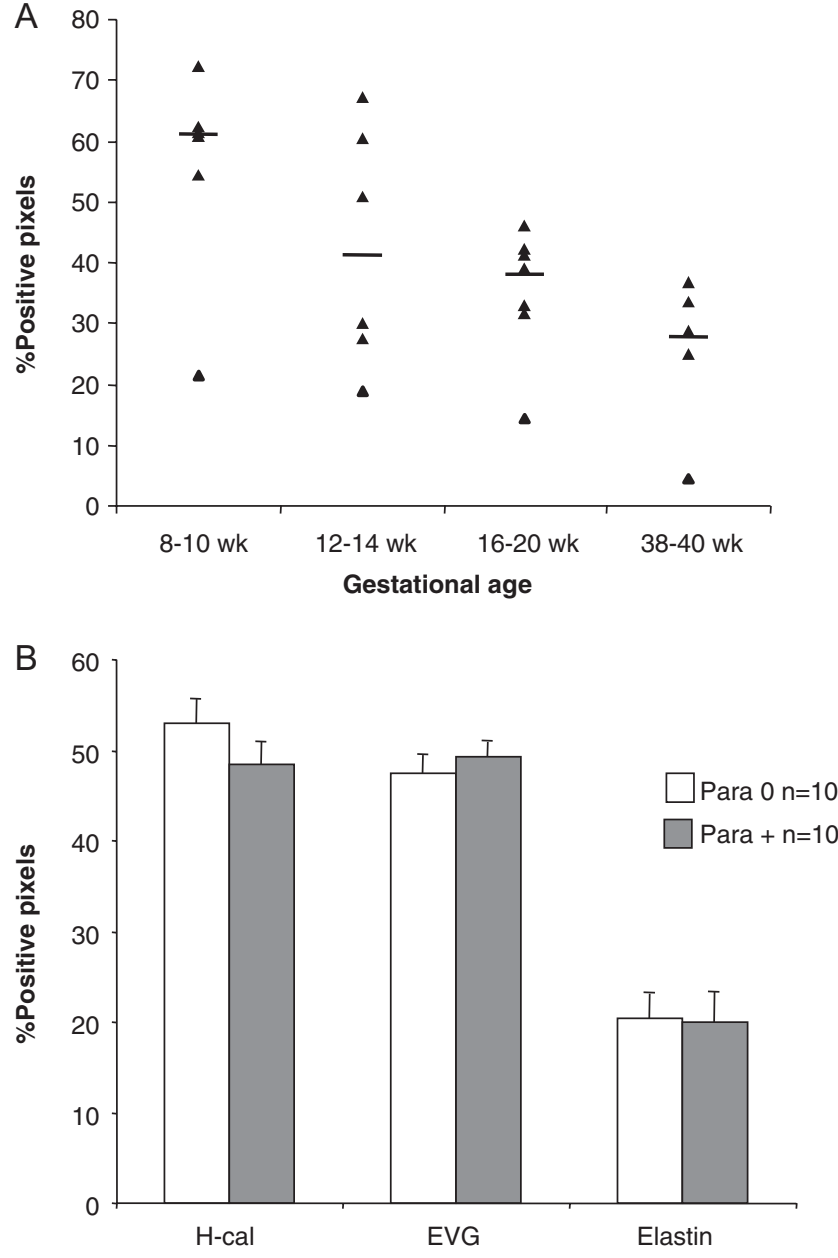

Figure 4 (A) Effect of gestational age on spiral artery vascular smooth muscle. Each data point represents the mean \% positive pixels calculated for each placental bed sample and bars show the median percentage of positive pixels. (B) Effect of parity on spiral artery smooth muscle stained for h-cal, and elastin detected with Elastic van Gieson stain and anti-elastin. Data are expressed as the percentage of positive pixels in the image of the vessel wall.

(P1; $n=90)$, late partially remodelled (P2; $n=90)$ and completely remodelled (C; $n=17)$; details of this classification have been published and illustrated previously (Lash et al. 2016). Another investigator (HAO) blinded to this classification analysed images of the vessels to determine any correlation between the percentage of positive pixels and the degree of remodelling.

No difference in the percentage of positive pixels was found between vessels in the groups subjectively assessed as $\mathrm{N}(55.46 \pm 2.32 \% ; n=33)$ and P1 (55.24 \pm 1.45 ; $n=90$ ). There was a significant difference between vessels in the two groups showing partial remodelling designated P1 and P2 $(42.93 \pm 1.52 ; n=90 ; P<0.0001)$ and between the groups $\mathrm{P} 2$ and $\mathrm{C}(14.05 \pm 2.47, n=17$; $P<0.0001$ ) (Fig. 5A).
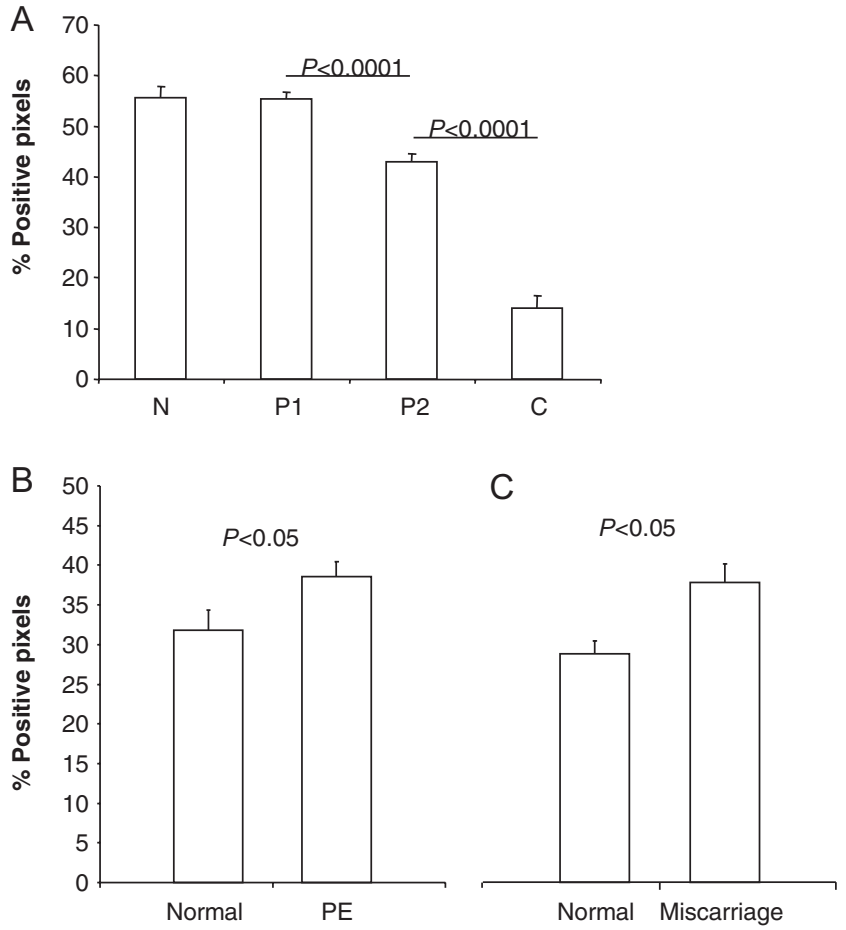

Figure 5 (A) Percentage positive staining for h-cal in non-transformed vessels $(\mathrm{N})$ compared with partially transformed (P1 and $\mathrm{P} 2)$ and completely transformed $(\mathrm{C})$ vessels. ( $\mathrm{B}$ and $\mathrm{C}$ ) Comparison of positive staining in spiral arteries from (B) 3rd trimester normal pregnancies and those complicated by pre-eclampsia; and (C) gestational age matched normal pregnancy and late miscarriage. ${ }^{*} P<0.05$.

\section{Pathological pregnancy}

All assessment of pathological samples and controls was blind to the identity of the samples.

\section{Pre-eclampsia}

A comparison of third trimester controls and women with PE is shown in Fig. 5B. Desmin immunoreactivity was significantly higher in SpAs from five subjects with PE $(38.6 \pm 1.7 ; n=15$ SpAs) compared with six normal subjects $(31.9 \pm 2.5 ; \mathrm{n}=18$ SpAs; $P<0.05)$.

\section{Late miscarriage}

Desmin immunostaining in spiral arteries from gestationally matched women undergoing elective pregnancy termination or late miscarriage at is shown in Fig. 5C. The percentage of positively stained pixels in SpAs from seven subjects after late miscarriage (38.1 \pm 2.4 , $n=39$ SpAs, $P<0.05)$ was significantly higher than in seven normal subjects $(29.0 \pm 1.6, n=64$ vessels).

\section{Discussion}

We have developed a simple, rapid and readily accessible technique for the quantification of $\mathrm{SpA}$ 
VSMCs based on pixel counting using Adobe Photoshop. This technique was highly reproducible for a single investigator on multiple occasions and there was good agreement between investigators. In addition, there was no difference in different cross-sections of the same vessel assessed in stepped sections from a paraffin block. Although demonstrated for VSMC quantification, this technique could be used to quantify other features of SpA remodelling, using appropriate immunohistochemistry or histochemical stains to detect different cell types, fibrinoid and elastica. For example, we have applied this technique to quantify elastic fibres in placental bed biopsies from nulliparous and parous women using immunostaining for elastic and the EVG stain.

VSMCs express different antigens depending on their differentiation state. In the current study we tested several different VSMC markers, including $\alpha S M A$ which is one of the first markers to appear and is also expressed by myofibroblasts. In our hands, h-caldesmon gave the highest staining intensity with minimal background reactivity, allowing for greatest ease and accuracy in analysis. Although h-caldesmon is expressed late in VSMC differentiation, it is a commonly used marker of muscle cells which has been reported to show more widespread expression in smooth muscle tumours than desmin (Ceballos et al. 2000, Nucci et al. 2001).

It is generally accepted that the quantity of VSMC in the wall of SpAs is reduced as the process of remodelling advances and the reduction in smooth muscle in myometrial SpAs with increasing gestation provided a means to validate the current method. As expected there was a significant reduction in immunostaining for h-caldesmon (as determined by percentage pixel number) with increasing gestational age.

Assessment of SpA remodelling often relies on subjective observations of one or more investigators. SpAs have been classified as partially, completely or nonremodelled and some investigators have further stratified partially remodelled SpAs based on the extent of muscle disorganisation, quantity of intramural trophoblast and the extent of fibrinoid deposition (Craven et al. 1998, Smith et al. 2009). Although the categories of completely and non-remodelled SpAs are relatively robust, partially transformed SpAs have a range of appearances, and this may account for differing views on the ubiquity or otherwise of failed SpA remodelling in the placental bed in pre-eclampsia. The use of a quantitative approach would allow objective assessment of SpA changes and potentially better definition of histological changes in pregnancy complications. The current approach allows quantification of various components of SpA remodelling and assessment of these may lead to better understanding of their inter-relationships. When we applied this technique to a group of arteries previously categorised as showing no remodelling, complete remodelling or early or late partial remodelling (Lash et al. 2016), we found significant differences between two subjective partial categories which would allow further separation of remodelling changes. However, we did not find any difference between non-remodelled and early partially remodelled vessels although morphologically vessels within these classifications look different. These vessels are often dilated with a thinner wall which shows separation of VSMCs, although the amount of vascular smooth muscle may remain unchanged. This suggests that some morphological assessment is still required or alternatively, a different parameter requires assessment to detect this stage of $\mathrm{SpA}$ remodelling.

SpAs 'spiral' within the tissue and are seen in several cross-sections within a given section. Measurement of diameter requires a transverse section of the artery. In previous studies arteries sectioned longitudinally (assessed by 'slant factor') were not included (Starzyk et al. 1997, Morgan et al. 1999). The current technique depends on assessment of the percentage of positive immunostained pixels within the area of the entire artery wall, in relation to the total number of pixels of the selected SpA. While in the current study we ensured that assessed arteries appeared symmetrical, we propose that some degree of slant in the section would not significantly alter the results. When the same artery was investigated at six different levels within a sample, the cross-sectional shape of the artery varied between sections at different levels, but this did not significantly alter the results obtained (Fig. 3C). Nevertheless, we did not specifically investigate this aspect of the method and a further validation may be required.

Ball et al. (2006a) performed a semi-quantitative assessment of various features of SpA remodelling and documented reduced endovascular and intramural trophoblast and fibrinoid deposition in myometrial SpAs in late miscarriage, although no change was seen in the arterial media using broad categories (disorganisation or no disorganisation). In the current study, increased smooth muscle (as evidenced by an increase in the percentage of immunopositive pixels) was detected in SpAs in late miscarriage compared with normal pregnancy using a subset of the samples used for the previous study (Ball et al. 2006a). This technique therefore allows detection of alterations in SpA remodelling, which were not recognised using a semiquantitative assessment of histological features. Further validation of the methodology was demonstrated using archived samples from pregnancies complicated by preeclampsia (Lyall et al. 2001, 2013). Using subjective criteria we had reported a decrease in the degree of SpA remodelling in this cohort of samples, a result validated in the current study as evidenced by an increase in the percentage of immunopositive pixels in the same immunostained sections.

Pre-eclampsia is most common in primigravidae (Macgillivray 1958, Chesley 1984), with a reduced incidence in subsequent pregnancies. We hypothesised that previous pregnancy may lead to reduced VSMC 
and elastica in myometrial SpAs, rendering them more susceptible to pregnancy-induced changes in subsequent pregnancies. There was, however, no difference in VSMC or elastic content between nulliparous and multiparous women. This contrasts with a recent study reporting persistent alterations in SpA architecture following pregnancy. Khong et al. (2003) studied SpAs within one low power field of the endomyometrial border in non-pregnant hysterectomy specimens from 28 women ranging from nulliparous to para 6. Muscular and non-muscular components were distinguished using a Masson trichrome stain and quantification was with an image analysis programme. Fragmentation and reduplication of the internal elastic lamina was assessed semi-quantitatively in orceinstained sections. There was increased duplication and fragmentation of the internal elastic lamina with increasing parity, with a significant difference between 'nulliparity' and 'multiparity'. There was also a trend to an increased proportion of non-muscular tissue with increasing parity. Furthermore, using uterine artery Doppler, Prefumo et al. (2004) demonstrated reduced mean resistance index in the second trimester in parous compared with nulliparous women. Endovascular trophoblast was also noted in a higher proportion of decidual SpAs from pregnancy terminations at 10-14 weeks gestation in parous compared with nulliparous women (Prefumo et al. 2006). It was suggested that these alterations may contribute to the protection from preeclampsia attributed to more than one viable pregnancy.

The explanation for the differences in our findings compared with those of Khong et al. (2003) is unclear. Although we focused on myometrial arteries, our analysis was of small placental bed samples, whereas Khong et al. (2003) examined arteries within superficial myometrium in hysterectomy samples. We chose an early gestational age and investigated only myometrial vessels which were not associated with extravillous trophoblast, to ensure that any changes observed could not be attributed to trophoblast-mediated effects in the current pregnancy. However, initial trophoblastindependent changes in SpAs in early pregnancy have been described, including separation of VSMCs (Craven et al. 1998, Pijnenborg et al. 2006). Although descriptions of such alterations have focused mainly on decidual SpAs, leading to the term 'decidua-associated remodelling' (Pijnenborg et al. 2006), similar changes may occur in myometrium leading to an apparent reduction in the relative area of the artery occupied by VSMCs. Although we were unable to detect any differences in the extent of elastic, we did not examine fragmentation of the internal elastic lamina.

In summary, we have developed a highly reproducible, simple and inexpensive technique for quantifying changes in SpA VSMCs, which could be applied to other components of SpA remodelling. We demonstrated a reduction in SpA VSMC content with increasing gestational age and increase in preeclampsia and late miscarriage. Although not replacing detailed morphological assessment and more complex morphometric techniques, this approach will allow more objective characterisation of different components of deficient SpA remodelling.

\section{Supplementary data}

This is linked to the online version of the paper at https://doi.org/10.1530/REP-19-0133.

\section{Declaration of interest}

The authors declare that there is no conflict of interest that could be perceived as prejudicing the impartiality of the research reported.

\section{Funding}

This project was supported by funding from BBSRC (S19967 and BB/E016790/1).

\section{Author contribution statement}

$\mathrm{H}$ A O performed the experiments, data analysis and wrote the manuscript; B A I performed experiments; G E L conceived the study, performed analysis and edited the manuscript; B S performed experiments; E B collected samples; R F S conceived the study; S C R collected samples; J N B conceived the study and edited the manuscript.

\section{Acknowledgements}

The authors are grateful to all the patients who provided samples for the study and to the staff of the Royal Victoria Infirmary, Newcastle upon Tyne for assistance in collection of samples.

\section{References}

Abberton KM, Taylor NH, Healy DL \& Rogers PA 1996 Vascular smooth muscle alpha-actin distribution around endometrial arterioles during the menstrual cycle: increased expression during the perimenopause and lack of correlation with menorrhagia. Human Reproduction $11204-$ 211. (https://doi.org/10.1093/oxfordjournals.humrep.a019020)

Abberton KM, Healy DL \& Rogers PA 1999 Smooth muscle alpha actin and myosin heavy chain expression in the vascular smooth muscle cells surrounding human endometrial arterioles. Human Reproduction $\mathbf{1 4}$ 3095-3100. (https://doi.org/10.1093/humrep/14.12.3095)

Ball E, Bulmer JN, Ayis S, Lyall F \& Robson SC 2006a Late sporadic miscarriage is associated with abnormalities in spiral artery transformation and trophoblast invasion. Journal of Pathology 208 535-542. (https://doi. org/10.1002/path.1927)

Ball E, Robson SC, Ayis S, Lyall F \& Bulmer JN 2006b Early embryonic demise: no evidence of abnormal spiral artery transformation or trophoblast invasion. Journal of Pathology 208 528-534. (https://doi. org/10.1002/path.1926) 
Ceballos KM, Nielsen GP, Selig MK \& O'Connell JX 2000 Is anti-hcaldesmon useful for distinguishing smooth muscle and myofibroblastic tumors? An immunohistochemical study. American Journal of Clinical Pathology 114 746-753. (https://doi.org/10.1309/K5JP-A9EN-UWN7B5GG)

Chesley LC 1984 History and epidemiology of preeclampsia-eclampsia. Clinical Obstetrics and Gynecology 27 801-820. (https://doi. org/10.1097/00003081-198412000-00004)

Craven CM, Morgan T \& Ward K 1998 Decidual spiral artery remodelling begins before cellular interaction with cytotrophoblasts. Placenta 19 241-252. (https://doi.org/10.1016/S0143-4004(98)90055-8)

Khong TY, De Wolf F, Robertson WB \& Brosens I 1986 Inadequate maternal vascular response to placentation in pregnancies complicated by pre-eclampsia and by small-for-gestational age infants. British Journal of Obstetrics and Gynaecology 93 1049-1059. (https://doi. org/10.1111/j.1471-0528.1986.tb07830.x)

Khong TY, Adema ED \& Erwich JJ 2003 On an anatomical basis for the increase in birth weight in second and subsequent born children. Placenta 24 348-353. (https://doi.org/10.1053/plac.2002.0922)

Kim YM, Bujold E, Chaiworapongsa T, Gomez R, Yoon BH, Thaler HT, Rotmensch S \& Romero R 2003 Failure of physiologic transformation of the spiral arteries in patients with preterm labor and intact membranes. American Journal of Obstetrics and Gynecology 189 1063-1069. (https://doi.org/10.1067/s0002-9378(03)00838-x)

Lash GE, Pitman H, Morgan HL, Innes BA, Agwu CN \& Bulmer JN 2016 Decidual macrophages: key regulators of vascular remodeling in human pregnancy. Journal of Leukocyte Biology 100 315-325. (https://doi. org/10.1189/jlb.1A0815-351R)

Lehr HA, Jacobs TW, Yaziji H, Schnitt SJ \& Gown AM 2001 Quantitative evaluation of HER-2/neu status in breast cancer by fluorescence in situ hybridization and by immunohistochemistry with image analysis. American Journal of Clinical Pathology 115 814-822. (https://doi. org/10.1309/AJ84-50AK-1X1B-1Q4C)

Lyall F, Simpson H, Bulmer JN, Barber A \& Robson SC 2001 Transforming growth factor beta expression in human placenta and placental bed in the third trimester normal pregnancy, preeclampsia, and fetal growth restriction. American Journal of Pathology 159 1827-1838. (https://doi. org/10.1016/s0002-9440(10)63029-5)

Lyall F, Robson SC \& Bulmer JN 2013 Spiral artery remodeling and trophoblast invasion in preeclampsia and fetal growth restriction: relationship to clinical outcome. Hypertension 62 1046-1054. (https:// doi.org/10.1161/HYPERTENSIONAHA.113.01892)

MacGillivray I 1958 Some observations on the incidence of pre-eclampsia. Journal of Obstetrics and Gynaecology for the British Empire 65536 539. (https://doi.org/10.1111/j.1471-0528.1958.tb08557.x)

Matkowskyj KA, Cox R, Jensen RT \& Benya RV 2003 Quantitative immunohistochemistryby measuring cumulativesignal strengthaccurately measures receptor number. Journal of Histochemistry and Cytochemistry 51 205-214. (https://doi.org/10.1177/002215540305100209)

Meekins JW, Pijnenborg R, Hanssens M, McFadyen IR \& van Asshe A 1994 A study of placental bed spiral arteries and trophoblast invasion in normal and severe pre-eclamptic pregnancies. British Journal of Obstetrics and Gynaecology 101 669-674. (https://doi.org/10.1111/j.1471-0528.1994. tb13182.x)

Morgan T, Craven C, Lalouel JM \& Ward K 1999 Angiotensinogen Thr235 variant is associated with abnormal physiologic change of the uterine spiral arteries in first-trimester decidua. American Journal of Obstetrics and Gynecology $\mathbf{1 8 0} \quad 95-102 . \quad$ (https://doi.org/10.1016/s00029378(99)70156-0)

Nucci MR, O'Connell JT, Huettner PC, Cviko A, Sun D \& Quade BJ 2001 h-Caldesmon expression effectively distinguishes endometrial stromal tumors from uterine smooth muscle tumors. American Journal of Surgical Pathology 25 455-463. (https://doi.org/10.1097/00000478-20010400000004)

Owens GK 1995 Regulation of differentiation of vascular smooth muscle cells. Physiological Reviews 75 487-517. (https://doi.org/10.1152/ physrev.1995.75.3.487)

Pijnenborg R, Anthony J, Davey DA, Rees A, Tiltman A, Vercruysse L \& van Assche A 1991 Placental bed spiral arteries in the hypertensive disorders of pregnancy. British Journal of Obstetrics and Gynaecology 98 648-655. (https://doi.org/10.1111/j.1471-0528.1991.tb13450.x)

Pijnenborg R, Vercruysse L \& Hanssens M 2006 The uterine spiral arteries in human pregnancy: facts and controversies. Placenta 27 939-958. (https://doi.org/10.1016/j.placenta.2005.12.006)

Prefumo F, Bhide A, Sairam S, Penna L, Hollis B \& Thilaganathan B 2004 Effect of parity on second-trimester uterine artery Doppler flow velocity and waveforms. Ultrasound in Obstetrics and Gynecology 23 46-49. (https://doi.org/10.1002/uog.908)

Prefumo F, Ganapathy R, Thilaganathan B \& Sebire NJ 2006 Influence of parity on first trimester endovascular trophoblast invasion. Fertility and Sterility 85 1032-1036. (https://doi.org/10.1016/j.fertnstert.2005.09.055)

Price RJ, Owens GK \& Skalak TC 1994 Immunohistochemical identification of arteriolar development using markers of smooth muscle differentiation. Evidence that capillary arterialization proceeds from terminal arterioles. Circulation Research 75 520-527. (https://doi. org/10.1161/01.res.75.3.520)

Robson SC, Simpson H, Ball E, Lyall F \& Bulmer JN 2002 Punch biopsy of the human placental bed. American Journal of Obstetrics and Gynecology 187 1349-1355. (https://doi.org/10.1067/mob.2002.126866)

Rogers PA \& Abberton KM 2003 Endometrial arteriogenesis: vascular smooth muscle cell proliferation and differentiation during the menstrual cycle and changes associated with endometrial bleeding disorders. Microscopy Research and Technique 60 412-419. (https://doi.org/10.1002/ jemt.10279)

Skalli O, Ropraz P, Trzeciak A, Benzonana G, Gillessen D \& Gabbiani G 1986 A monoclonal antibody against alpha-smooth muscle actin: a new probe for smooth muscle differentiation. Journal of Cell Biology 103 2787-2796. (https://doi.org/10.1083/jcb.103.6.2787)

Smith SD, Dunk CE, Aplin JD, Harris LK \& Jones RL 2009 Evidence for immune cell involvement in decidual spiral arteriole remodeling in early human pregnancy. American Journal of Pathology 174 1959-1971. (https://doi.org/10.2353/ajpath.2009.080995)

Starzyk KA, Salafia CM, Pezzullo JC, Lage JM, Parkash V, Vercruysse L, Hanssens M \& Pijnenborg R 1997 Quantitative differences in arterial morphometry define the placental bed in pre-eclampsia. Human Pathology 28 353-358. (https://doi.org/10.1016/s0046-8177(97)90135-0)

Received 20 March 2019

First decision 11 April 2019

Revised manuscript received 6 August 2019

Accepted 27 August 2019 